Supporting Information for

\title{
Molecular Details of Acetate Binding to a New Diamine Receptor by NMR and FT-IR Analyses
}

Biswadip Banerji, ${ }^{*} \dagger$ Moumita Chatterjee, ${ }^{\dagger}$ Uttam Pal, ${ }^{\star}$ and Nakul Chandra

Maiti $^{*}+$

${ }^{\dagger}$ Organic \& Medicinal Chemistry Division and ${ }^{\star}$ Structural Biology \& Bioinformatics

Division, CSIR-Indian Institute of Chemical Biology, 4, Raja S.C. Mullick Road, Kolkata 700032, India

*Phone: +91-33-2499-5709. Fax: +91-33-2473-5197.

E-mail: biswadip.banerji@gmail.com. (B.B.)

*Phone: +91-33-2499-5940. Fax: +91-33-2473-5197.

E-mail: ncmaiti@iicb.res.in. (N.C.M.) 


\section{Contents}

1. Figure $S 1$ page $S 3$

2. Figure $\mathrm{S} 2$

page $\mathrm{S} 4$

3. Figure $\mathrm{S} 3$

page $\mathrm{S} 5$

4. Characterization of the receptor $\mathrm{R}$

page S6

5. ${ }^{13} \mathrm{C}$ NMR Spectra of receptor $\mathrm{R}$ in $\mathrm{DMSO}-\mathrm{d}_{6}$

page $\mathrm{S} 6$

6. ${ }^{1} \mathrm{H}$ NMR of receptor $\mathrm{R}$ in $\mathrm{DMSO}-\mathrm{d}_{6}$

page $\mathrm{S} 7$

7. ${ }^{1} \mathrm{H}$ NMR of receptor $\mathrm{R}+0.5$ equivalent TBOAc in DMSO- $\mathrm{d}_{6}$

page $S 7$

8. ${ }^{1} \mathrm{H}$ NMR of receptor $\mathrm{R}+1$ equivalent TBOAc in DMSO- $\mathrm{d}_{6}$

page $\mathrm{S} 7$

9. ${ }^{1} \mathrm{H}$ NMR of receptor $\mathrm{R}+2$ equivalent TBOAc in DMSO- $\mathrm{d}_{6}$

page $\mathrm{S} 8$

10. ${ }^{1} \mathrm{H}$ NMR of receptor $\mathrm{R}+3$ equivalent TBOAc in DMSO- $\mathrm{d}_{6}$

page $\mathrm{S} 8$

11. ${ }^{1} \mathrm{H}$ NMR of receptor $\mathrm{R}+5$ equivalent TBOAc in DMSO- $\mathrm{d}_{6}$

page $\mathrm{S} 8$

12. ${ }^{1} \mathrm{H}$ NMR of receptor $\mathrm{R}+7$ equivalent TBOAc in DMSO- $\mathrm{d}_{6}$

page $\mathrm{S} 9$

13. ${ }^{1} \mathrm{H}$ NMR of receptor $\mathrm{R}+2$ equivalent $\mathrm{TBBr}$ in $\mathrm{DMSO}-\mathrm{d}_{6}$

page $\mathrm{S} 9$

14. ${ }^{1} \mathrm{H}$ NMR of receptor $\mathrm{R}+4$ equivalent TBBr in DMSO- $\mathrm{d}_{6}$

page $S 9$

15. ${ }^{1} \mathrm{H}$ NMR of receptor $\mathrm{R}+2$ equivalent TBI in DMSO- $\mathrm{d}_{6}$

page $\mathrm{S} 10$

16. Optimized geometry of the acetate

page $\mathrm{S} 10$

17. Optimized geometry of the receptor

page $\mathrm{S} 11$

18. Input geometry of the complex

page $\mathrm{S} 12$

19. Optimized geometry of the complex

page $S 13$

20. Theoretical IR and Raman spectra of acetate

page $\mathrm{S} 14$

21. Theoretical IR and Raman spectra of the receptor

page $\mathrm{S} 14$

22. Theoretical IR and Raman spectra of the complex

page $S 15$ 


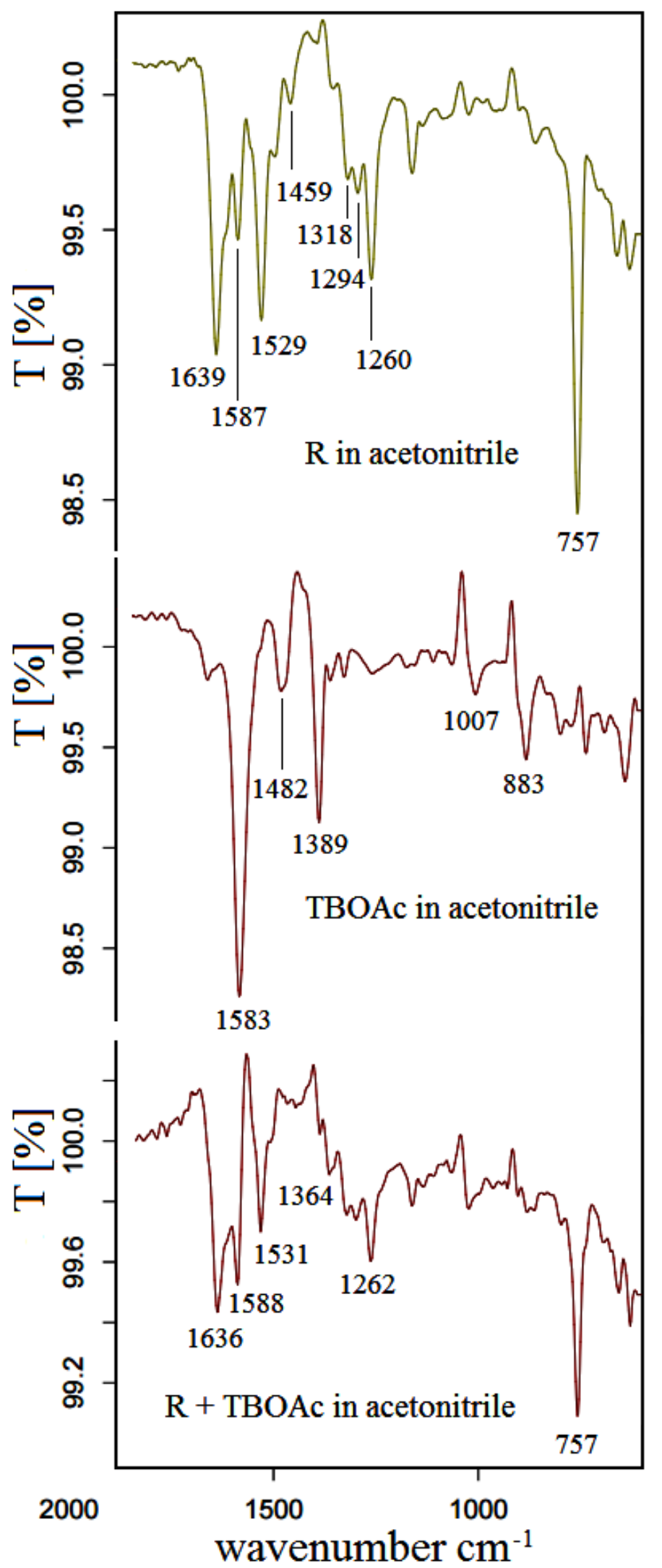

Figure S1: Fingerprint region of the FT-IR spectra of receptor $\mathbf{R}$ (top), TBOAc (middle) and the mixture of receptor $\mathbf{R}$ with TBOAc (bottom) in acetonitrile solvent. 


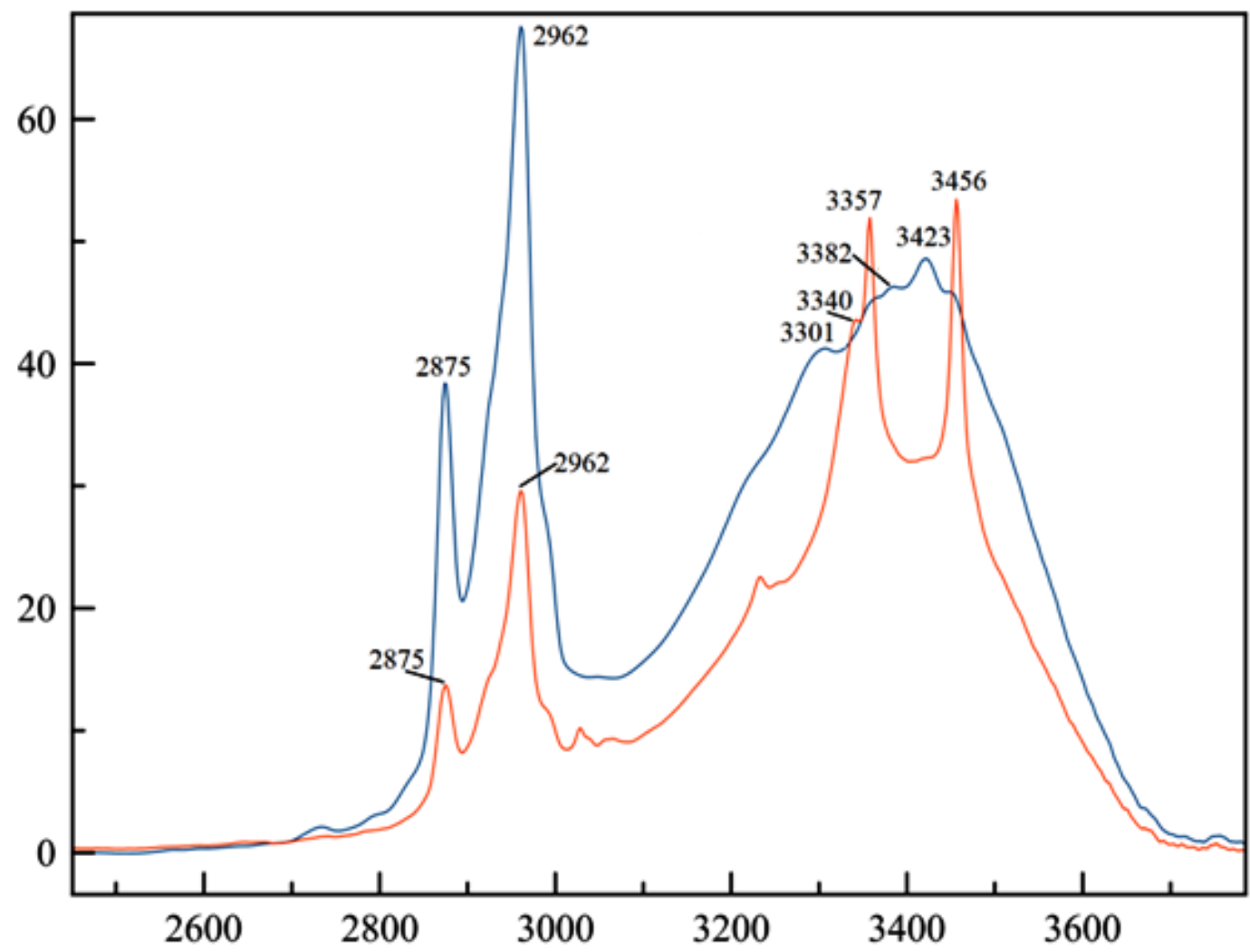

Figure S2. Partial IR spectrum (2800-3700 $\left.\mathrm{cm}^{-1}\right)$ of the mixture of receptor $\mathbf{R}$ and acetate anion shown in blue and simple addition of receptor $\mathbf{R}$ and acetate anion IR shown in red 


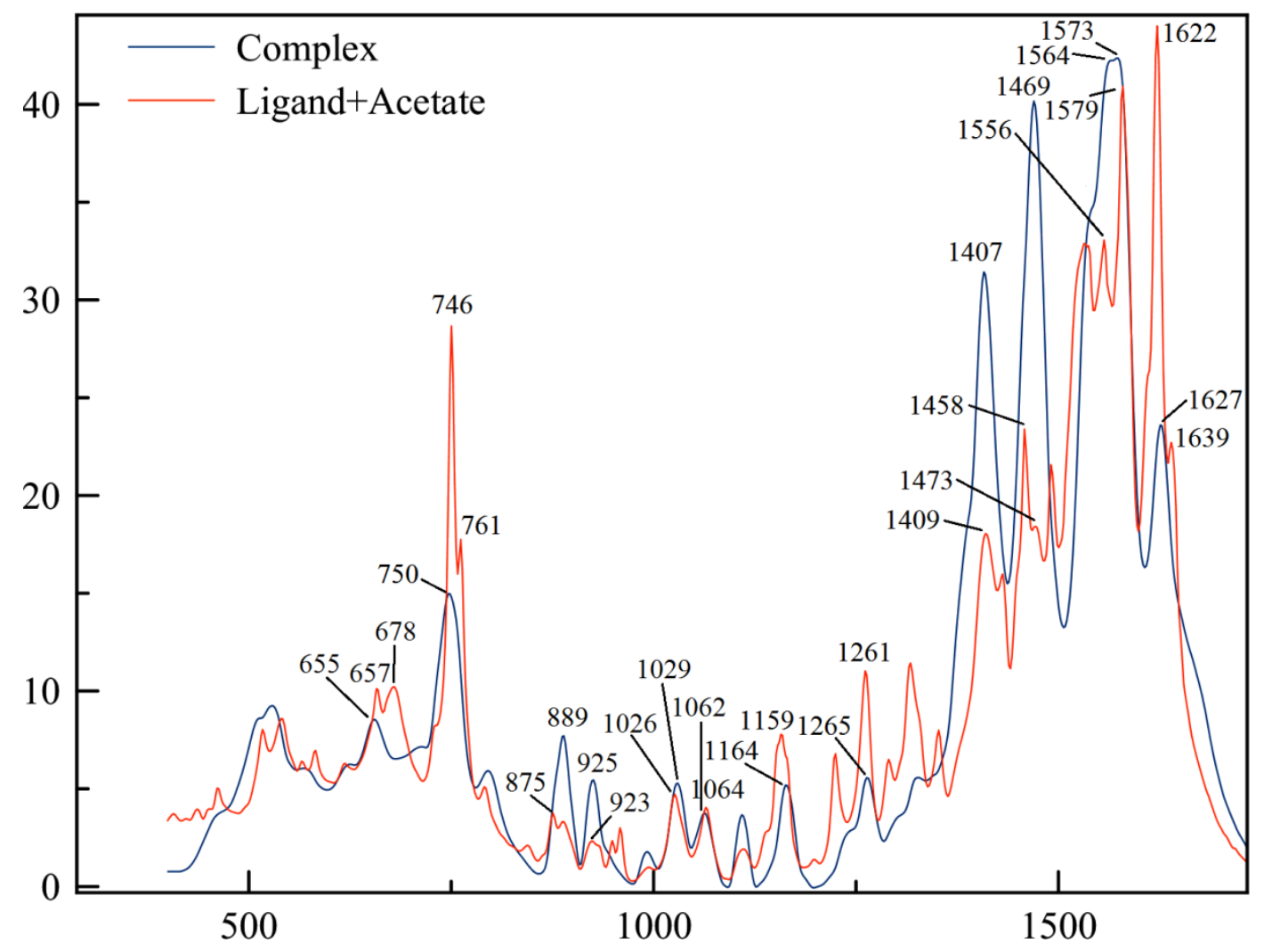

Figure S3: Finger print region of the IR spectrum of the mixture of receptor $\mathbf{R}$ and acetate anion shown in blue and simple addition of receptor $\mathbf{R}$ and acetate anion IR shown in red 


\section{Characterization of the receptor $\mathbf{R}$ :}

2-Amino-N-(2-amino-benzyl)-benzamide (receptor $\mathbf{R}$ ): White solid, $\mathrm{R}_{\mathrm{f}} 0.60$ (petroleum ether, ethyl acetate 3:2), Melting point $105{ }^{\circ} \mathrm{C},{ }^{1} \mathrm{H}$ NMR (300 MHz, DMSO-d 6 ): $\delta$ (in ppm) $4.26(2 \mathrm{H}, \mathrm{d}, J=6), 5.13(2 \mathrm{H}, \mathrm{s}), 6.41(2 \mathrm{H}, \mathrm{s}), 6.48-6.53(2 \mathrm{H}, \mathrm{m}), 6.615(1 \mathrm{H}, \mathrm{d}, J=9), 6.695$ $(1 \mathrm{H}, \mathrm{d}, J=9), 6.95(1 \mathrm{H}, \mathrm{t}, J=9), 7.02(1 \mathrm{H}, \mathrm{d}, J=6), 7.11-7.16(1 \mathrm{H}, \mathrm{m}), 7.50(1 \mathrm{H}, \mathrm{d}, J=6)$, $8.63(1 \mathrm{H}, \mathrm{t}, J=6) ;{ }^{13} \mathrm{C} \mathrm{NMR}\left(150 \mathrm{MHz}, \mathrm{CDCl}_{3}\right): \delta$ (in ppm) 40.35, 114.39, 114. 60, 115.72, $116.42,122.36,127.70,128.00,129.14,131.78,146.17,149.70,168.96 ;$ HRMS (EI+): m/z Calcd for $\mathrm{C}_{14} \mathrm{H}_{15} \mathrm{~N}_{3} \mathrm{O}(\mathrm{M})^{+} 241.1215$, Found: $\mathrm{m} / \mathrm{z}$ 241.1216. IR $\left(\mathrm{cm}^{-1}\right)$ 3456, 3354, 3232, 3031, $2941,1620,1582,1527,1455,1319,1296,1261,1225,1157,955,754,679,540$.

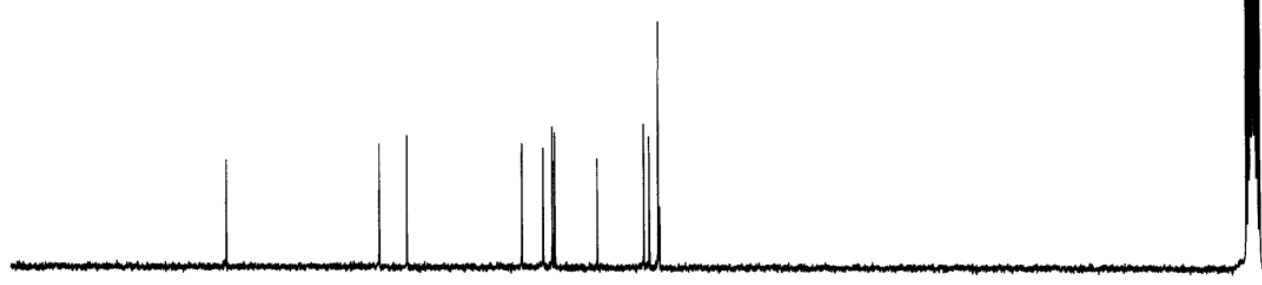

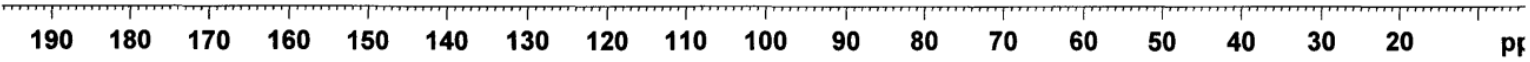

${ }^{13} \mathrm{C}$ NMR Spectra of receptor $\mathrm{R}$ in DMSO-d $\mathrm{d}_{6}$ 

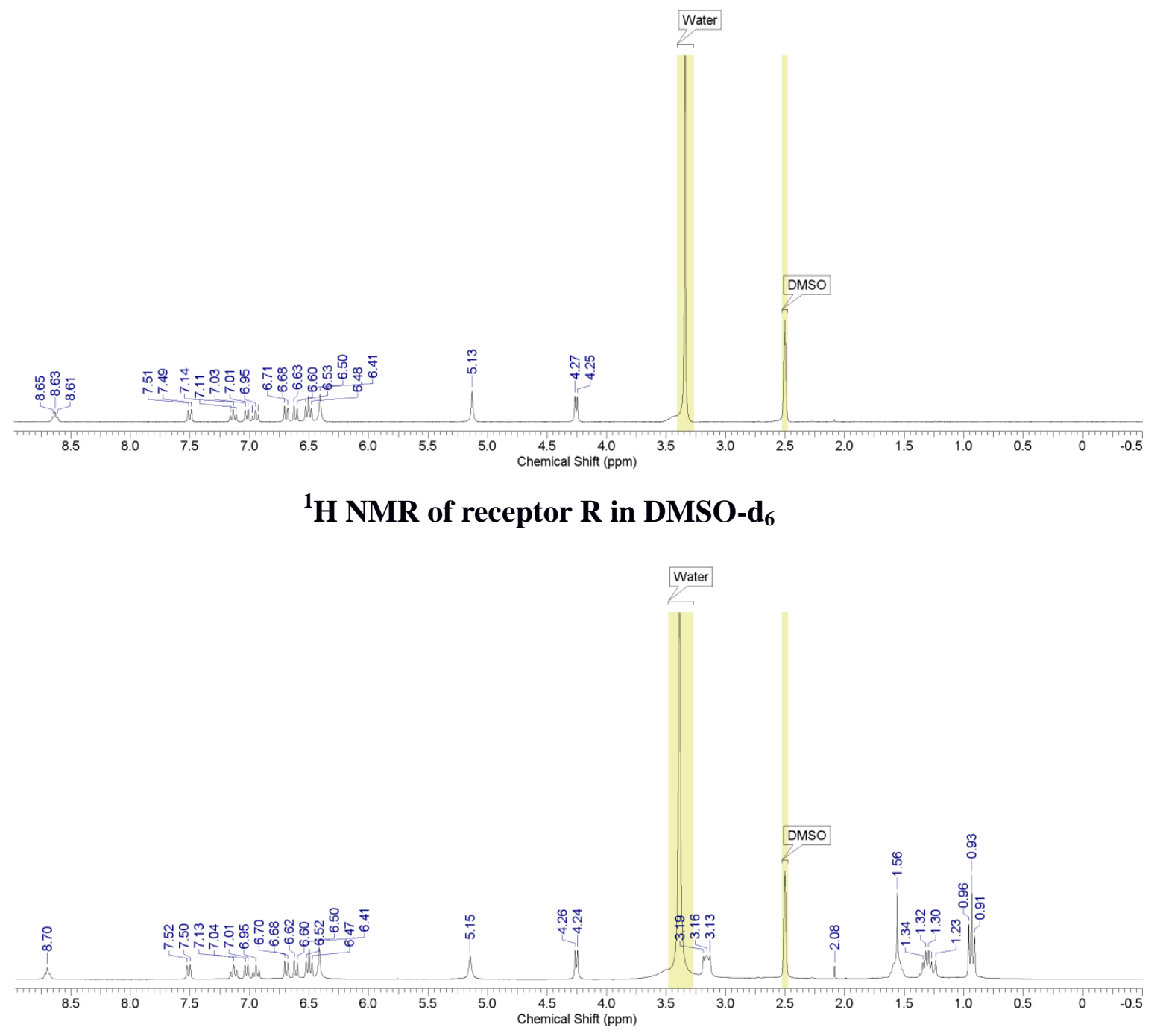

${ }^{1} \mathrm{H}$ NMR of receptor $\mathrm{R}+0.5$ equivalent TBOAc in DMSO-d $\mathrm{d}_{6}$

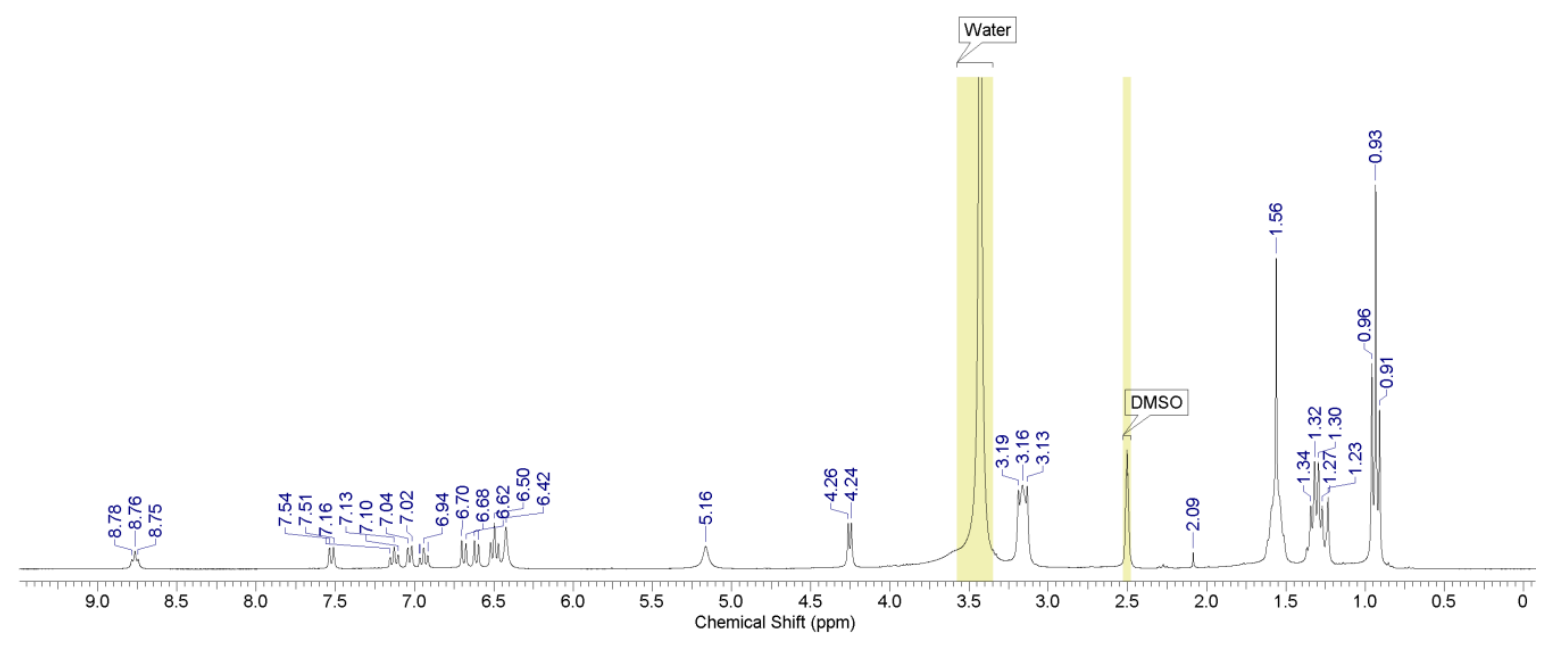

${ }^{1} \mathrm{H}$ NMR of receptor $\mathrm{R}+1$ equivalent TBOAc in DMSO-d 


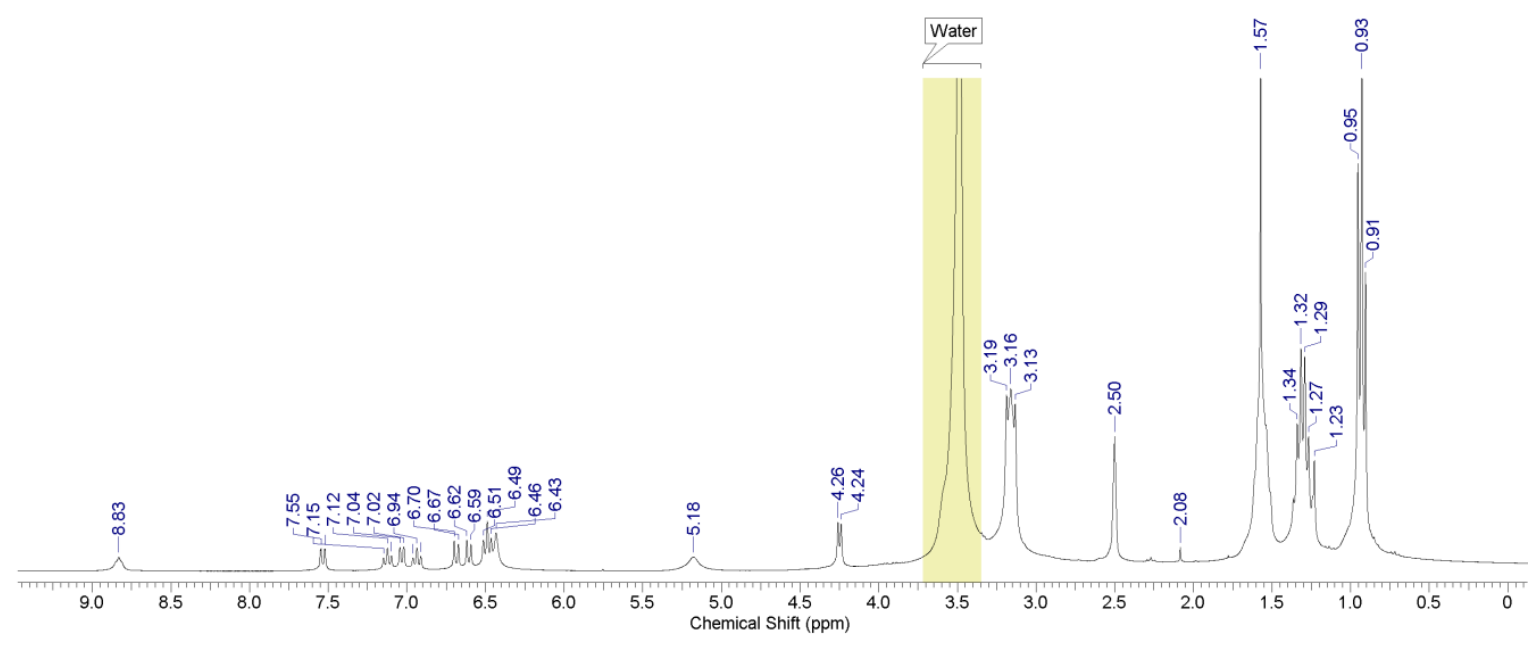

${ }^{1} H$ NMR of receptor $R+2$ equivalent TBOAc in DMSO-d ${ }_{6}$

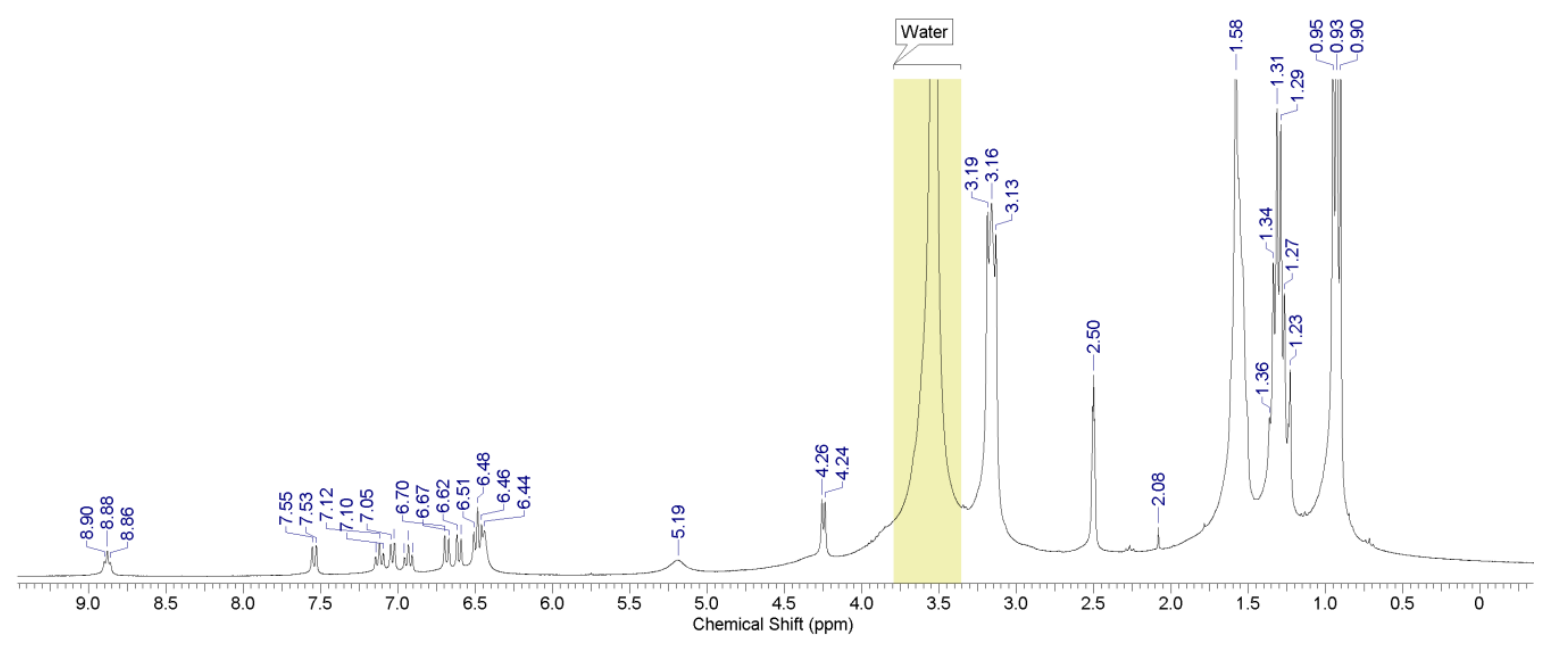

${ }^{1} \mathrm{H}$ NMR of receptor $\mathrm{R}+3$ equivalent TBOAc in DMSO-d $\mathrm{d}_{6}$

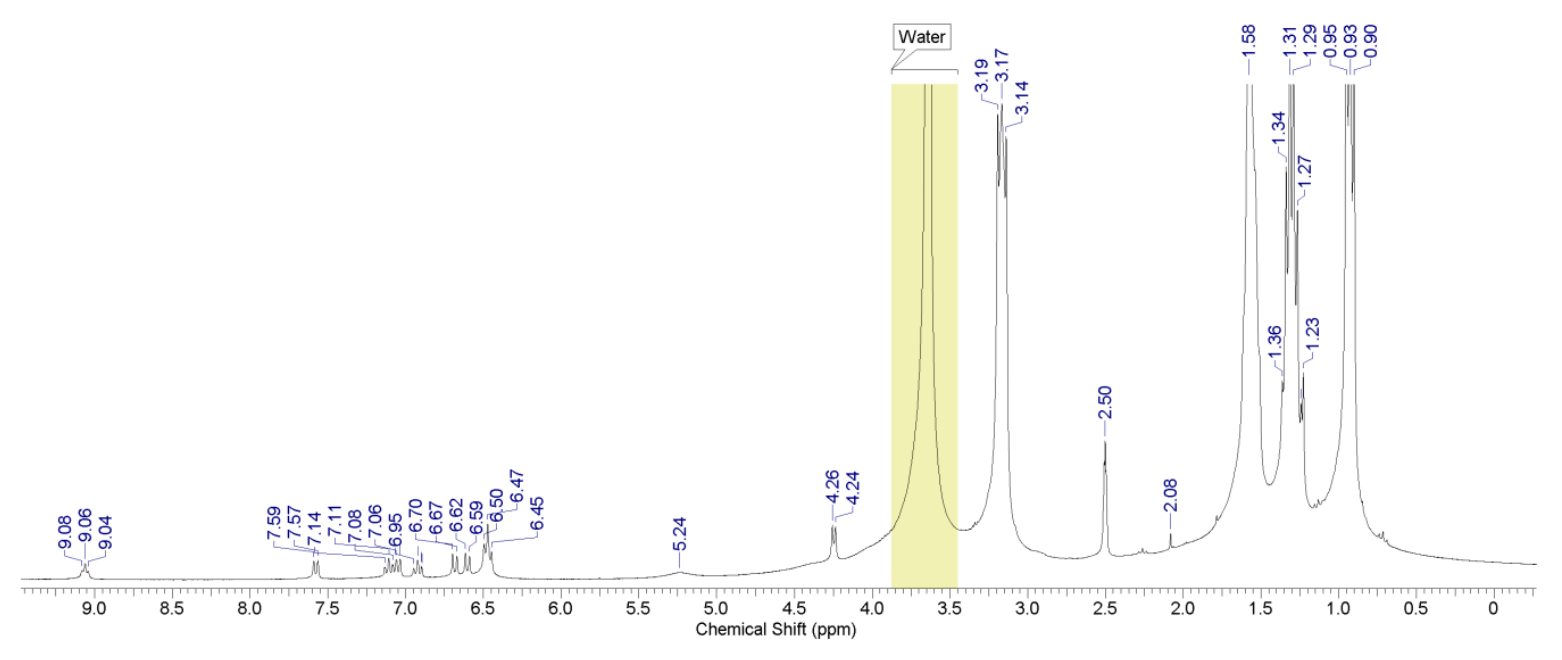

${ }^{1} \mathrm{H}$ NMR of receptor $\mathrm{R}+5$ equivalent TBOAc in DMSO-d $\mathrm{d}_{6}$ 


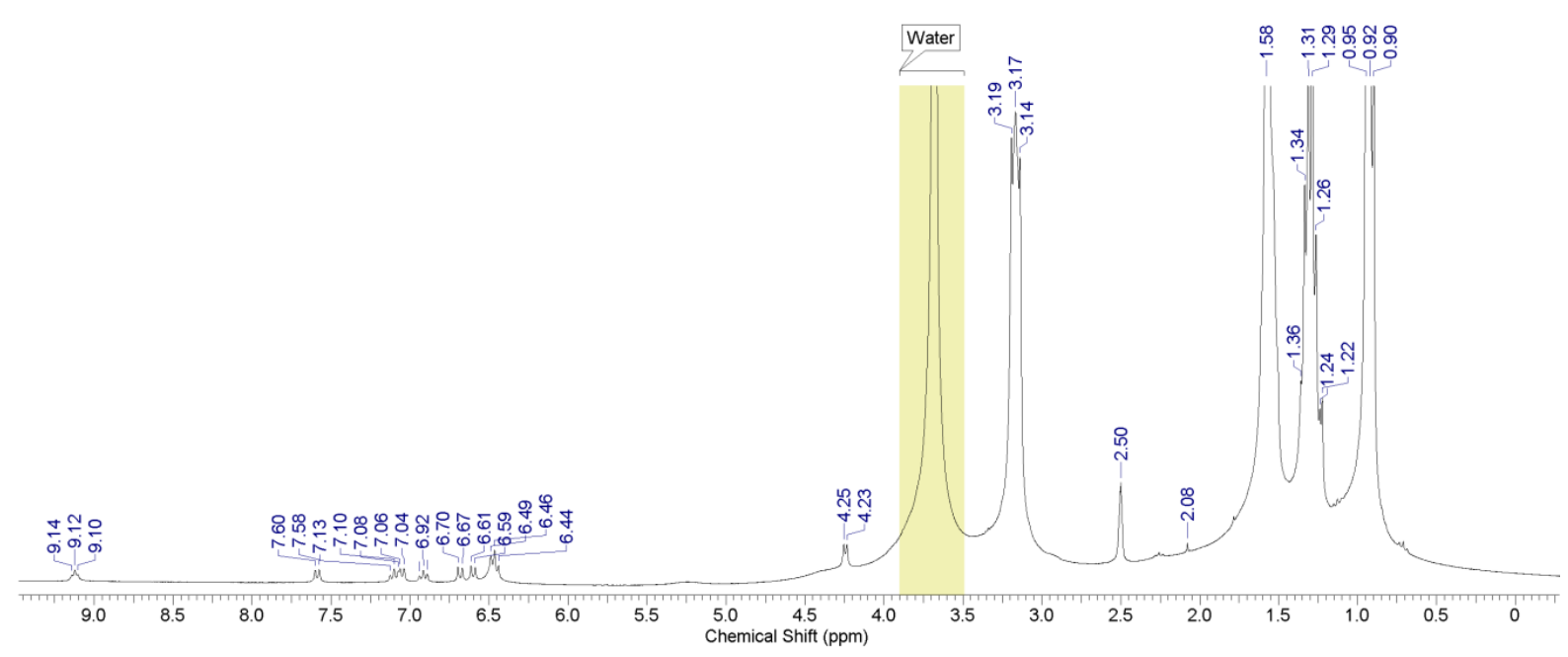

${ }^{1} \mathrm{H}$ NMR of receptor $\mathrm{R}+7$ equivalent TBOAc in DMSO-d $\mathrm{d}_{6}$

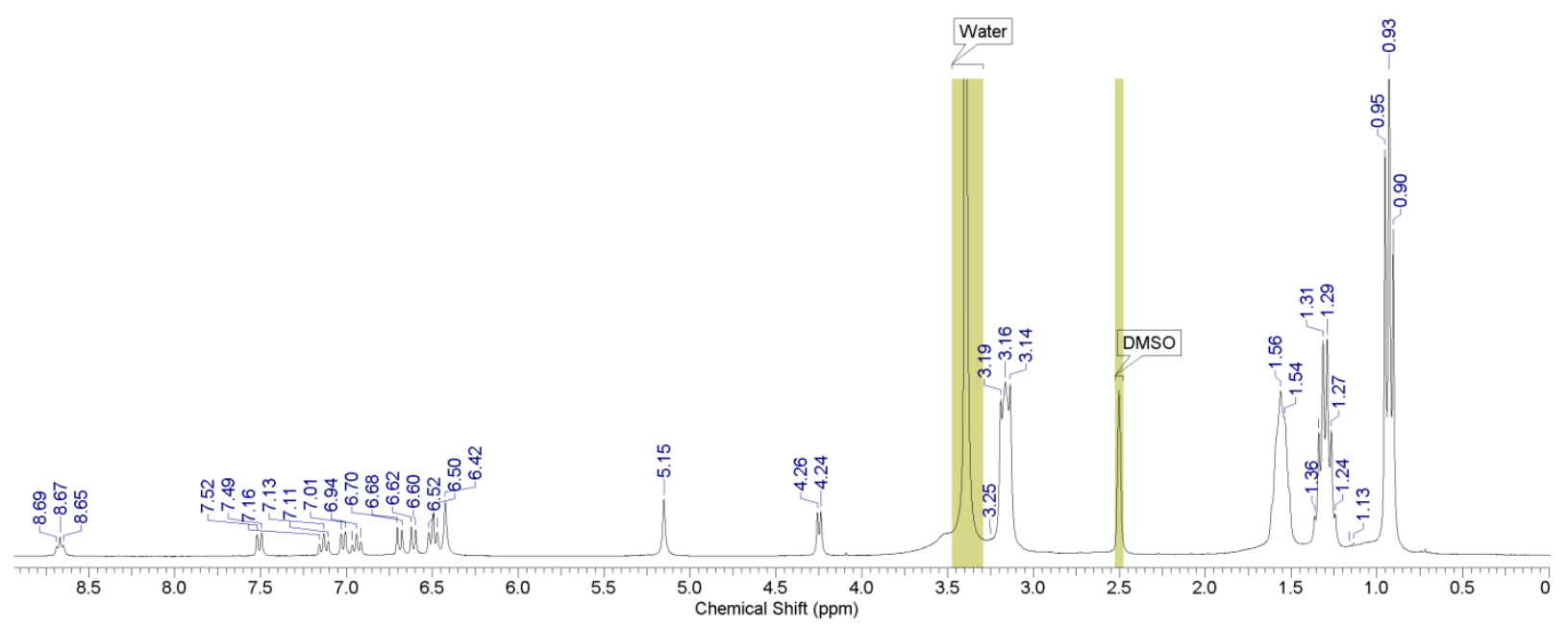

${ }^{1}$ H NMR of receptor $R+2$ equivalent TBBr in DMSO-d 6

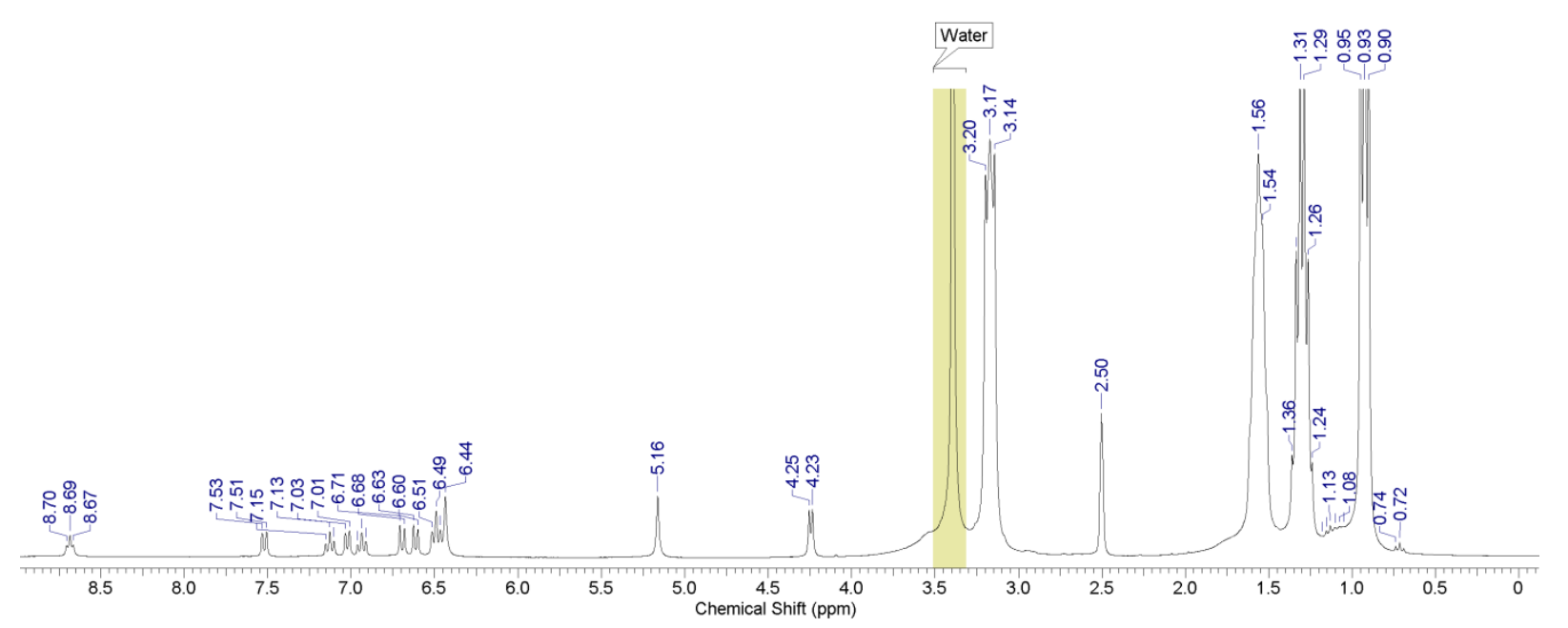

${ }^{1} \mathrm{H}$ NMR of receptor $\mathrm{R}+4$ equivalent $\mathrm{TBBr}$ in DMSO-d $\mathrm{d}_{6}$ 


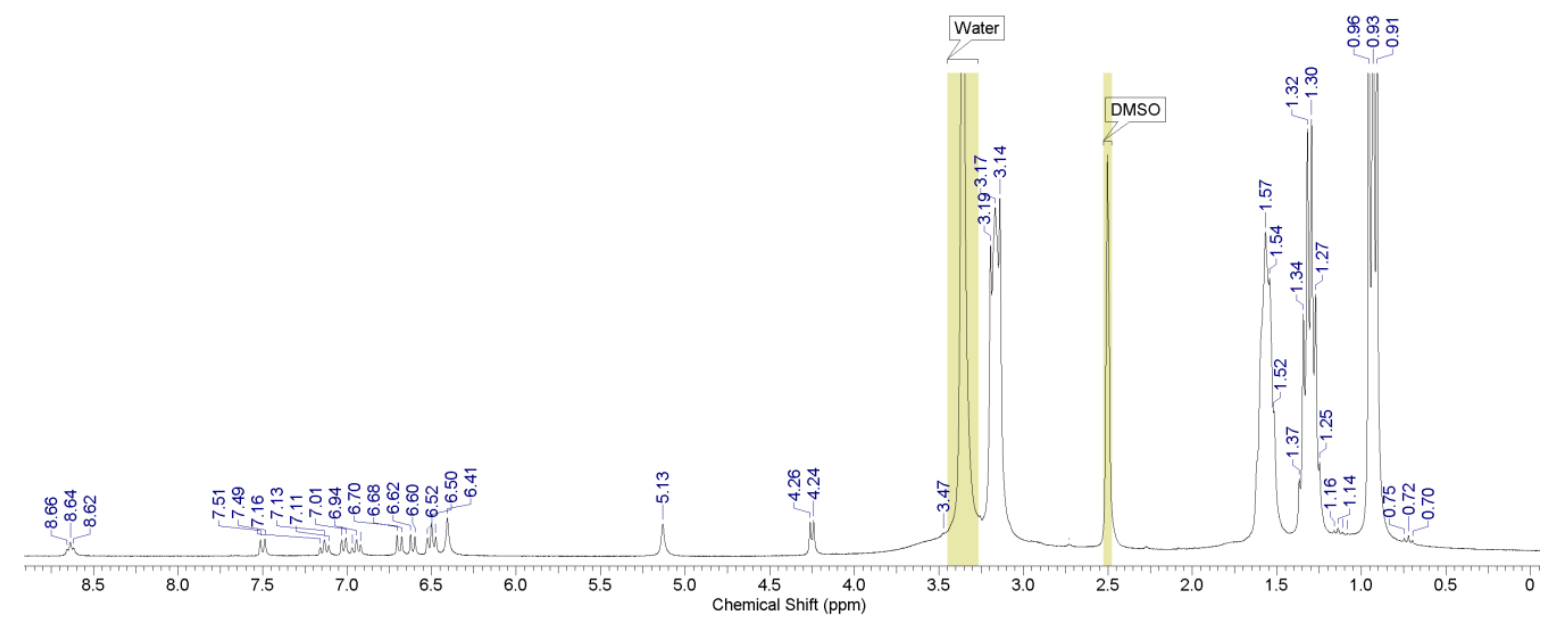

${ }^{1} \mathrm{H}$ NMR of receptor $\mathrm{R}+\mathbf{2}$ equivalent TBI in DMSO- $\mathrm{d}_{6}$

\section{Optimized geometry of the acetate}

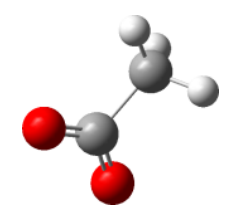

$E($ RB3LYP $)=-228.607030194$ Hartree/particle

Standard orientation:

\begin{tabular}{|c|c|c|c|c|c|}
\hline \multirow{2}{*}{$\begin{array}{l}\text { Center } \\
\text { Number }\end{array}$} & \multirow{2}{*}{$\begin{array}{l}\text { Atomic } \\
\text { Number }\end{array}$} & \multirow{2}{*}{$\begin{array}{c}\text { Atomic } \\
\text { Type }\end{array}$} & \multicolumn{3}{|c|}{ Coordinates (Angstroms) } \\
\hline & & & $\mathrm{X}$ & Y & Z \\
\hline 1 & 6 & 0 & -0.210068 & 0.001211 & -0.000356 \\
\hline 2 & 8 & 0 & -0.799957 & -1.106291 & 0.000068 \\
\hline 3 & 8 & 0 & -0.703518 & 1.155511 & 0.000069 \\
\hline 4 & 6 & 0 & 1.350190 & -0.047400 & -0.000193 \\
\hline 5 & 1 & 0 & 1.733576 & 0.490896 & -0.873153 \\
\hline 6 & 1 & 0 & 1.731307 & 0.467158 & 0.888068 \\
\hline 7 & 1 & 0 & 1.722183 & -1.074675 & -0.012719 \\
\hline
\end{tabular}




\section{Optimized geometry of the compound}

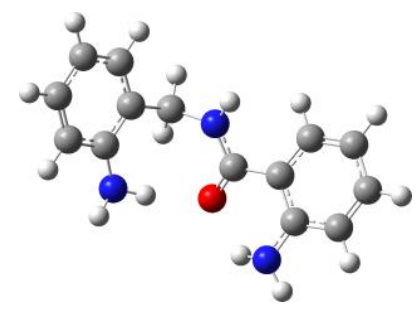

$E($ RB3LYP $)=-782.276497039$ Hartree/particle

Standard orientation:

\begin{tabular}{|c|c|c|c|c|c|}
\hline \multirow{2}{*}{$\begin{array}{l}\text { Center } \\
\text { Number }\end{array}$} & \multirow{2}{*}{$\begin{array}{l}\text { Atomic } \\
\text { Number }\end{array}$} & \multirow{2}{*}{$\begin{array}{c}\text { Atomic } \\
\text { Type }\end{array}$} & \multicolumn{3}{|c|}{ Coordinates (Angstroms) } \\
\hline & & & $\mathrm{X}$ & $\mathrm{Y}$ & Z \\
\hline & & & 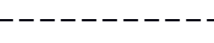 & --1 & --------- \\
\hline 1 & 8 & 0 & 0.670182 & 1.358638 & 1.220945 \\
\hline 2 & 7 & 0 & 2.734713 & 2.138564 & -0.340990 \\
\hline 3 & 1 & 0 & 2.021198 & 2.418190 & 0.320171 \\
\hline 4 & 1 & 0 & 3.483075 & 2.791523 & -0.507968 \\
\hline 5 & 7 & 0 & -0.064577 & -0.751880 & 0.879543 \\
\hline 6 & 1 & 0 & 0.073981 & -1.598633 & 0.352420 \\
\hline 7 & 7 & 0 & -2.100574 & 1.982661 & 0.240320 \\
\hline 8 & 1 & 0 & -2.205814 & 2.761851 & -0.390471 \\
\hline 9 & 1 & 0 & -1.157988 & 1.882299 & 0.605315 \\
\hline 10 & 6 & 0 & 2.198973 & -0.181998 & 0.211163 \\
\hline 11 & 6 & 0 & 3.069164 & 0.806398 & -0.319581 \\
\hline 12 & 6 & 0 & 4.287243 & 0.387479 & -0.887995 \\
\hline 13 & 1 & 0 & 4.946709 & 1.138349 & -1.309894 \\
\hline 14 & 6 & 0 & 4.653156 & -0.942875 & -0.910286 \\
\hline 15 & 1 & 0 & 5.603077 & -1.226736 & -1.348242 \\
\hline 16 & 6 & 0 & 3.816988 & -1.916314 & -0.360819 \\
\hline 17 & 1 & 0 & 4.110118 & -2.958001 & -0.352538 \\
\hline 18 & 6 & 0 & 2.610368 & -1.523083 & 0.188395 \\
\hline 19 & 1 & 0 & 1.982508 & -2.276268 & 0.648971 \\
\hline 20 & 6 & 0 & 0.890228 & 0.209925 & 0.804999 \\
\hline 21 & 6 & 0 & -1.383567 & -0.557397 & 1.493311 \\
\hline 22 & 1 & 0 & -1.301667 & 0.313448 & 2.142621 \\
\hline 23 & 1 & 0 & -1.580483 & -1.422824 & 2.130033 \\
\hline 24 & 6 & 0 & -2.514989 & -0.407243 & 0.501959 \\
\hline 25 & 6 & 0 & -3.298529 & -1.513734 & 0.178759 \\
\hline 26 & 1 & 0 & -3.082010 & -2.461141 & 0.663456 \\
\hline 27 & 6 & 0 & -4.345876 & -1.436754 & -0.731202 \\
\hline 28 & 1 & 0 & -4.939278 & -2.311859 & -0.963501 \\
\hline 29 & 6 & 0 & -4.618978 & -0.209963 & -1.329257 \\
\hline 30 & 1 & 0 & -5.431452 & -0.120158 & -2.041173 \\
\hline 31 & 6 & 0 & -3.861199 & 0.907614 & -1.018303 \\
\hline 32 & 1 & 0 & -4.090200 & 1.861310 & -1.482602 \\
\hline 33 & 6 & 0 & -2.799678 & 0.836970 & -0.102190 \\
\hline
\end{tabular}




\section{Input geometry of the complex}

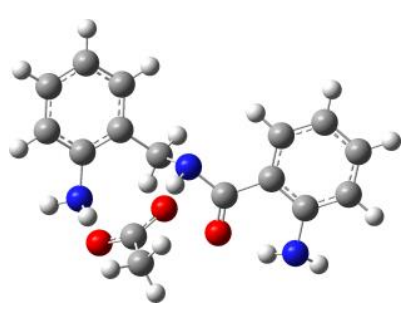

Input orientation:

\begin{tabular}{|c|c|c|c|c|c|}
\hline \multirow{2}{*}{$\begin{array}{l}\text { Center } \\
\text { Number }\end{array}$} & \multirow{2}{*}{$\begin{array}{l}\text { Atomic } \\
\text { Number }\end{array}$} & \multirow{2}{*}{$\begin{array}{c}\text { Atomic } \\
\text { Type }\end{array}$} & \multicolumn{3}{|c|}{ Coordinates (Angstroms) } \\
\hline & & & $\mathrm{X}$ & $\mathrm{Y}$ & Z \\
\hline & & & & -1 & ---- \\
\hline 1 & 8 & 0 & 0.000000 & 0.000000 & 0.000000 \\
\hline 2 & 7 & 0 & 0.000000 & 0.000000 & 2.474577 \\
\hline 3 & 1 & 0 & 0.583902 & 0.000000 & 1.662549 \\
\hline 4 & 1 & 0 & 0.577430 & 0.103993 & 3.290760 \\
\hline 5 & 7 & 0 & -1.467364 & 1.225095 & -1.445359 \\
\hline 6 & 1 & 0 & -1.859232 & 0.332676 & -1.742615 \\
\hline 7 & 7 & 0 & -0.137818 & -0.153234 & -4.427639 \\
\hline 8 & 1 & 0 & 0.311375 & -0.429802 & -5.265114 \\
\hline 9 & 1 & 0 & -0.672384 & -0.927653 & -4.078985 \\
\hline 10 & 6 & 0 & -1.407814 & 1.582724 & 1.144124 \\
\hline 11 & 6 & 0 & -0.940252 & 1.134609 & 2.394722 \\
\hline 12 & 6 & 0 & -1.385159 & 1.754791 & 3.574261 \\
\hline 13 & 1 & 0 & -1.021524 & 1.422458 & 4.528281 \\
\hline 14 & 6 & 0 & -2.287609 & 2.824125 & 3.509030 \\
\hline 15 & 1 & 0 & -2.629357 & 3.296135 & 4.411169 \\
\hline 16 & 6 & 0 & -2.739627 & 3.282349 & 2.260428 \\
\hline 17 & 1 & 0 & -3.424753 & 4.103211 & 2.212302 \\
\hline 18 & 6 & 0 & -2.278472 & 2.683168 & 1.075161 \\
\hline 19 & 1 & 0 & -2.605094 & 3.055146 & 0.127285 \\
\hline 20 & 6 & 0 & -0.918991 & 0.856700 & -0.130345 \\
\hline 21 & 6 & 0 & -0.497901 & 1.801038 & -2.355584 \\
\hline 22 & 1 & 0 & 0.380351 & 1.181904 & -2.451142 \\
\hline 23 & 1 & 0 & -0.207457 & 2.745192 & -1.938833 \\
\hline 24 & 6 & 0 & -1.145321 & 1.999372 & -3.752069 \\
\hline 25 & 6 & 0 & -1.893614 & 3.157574 & -4.073893 \\
\hline 26 & 1 & 0 & -2.008435 & 3.945036 & -3.362175 \\
\hline 27 & 6 & 0 & -2.512397 & 3.259635 & -5.340810 \\
\hline 28 & 1 & 0 & -3.095192 & 4.124908 & -5.585691 \\
\hline 29 & 6 & 0 & -2.368197 & 2.234486 & -6.282226 \\
\hline 30 & 1 & 0 & -2.842886 & 2.309785 & -7.242613 \\
\hline 31 & 6 & 0 & -1.592709 & 1.114392 & -5.970720 \\
\hline 32 & 1 & 0 & -1.471653 & 0.334488 & -6.687261 \\
\hline 33 & 6 & 0 & -0.976090 & 1.010908 & -4.719119 \\
\hline 34 & 6 & 0 & -2.338235 & -1.945165 & -2.641448 \\
\hline 35 & 8 & 0 & -1.437338 & -2.036819 & -3.579893 \\
\hline 36 & 8 & 0 & -2.601342 & -0.887245 & -1.934706 \\
\hline 37 & 6 & 0 & -3.195544 & -3.159170 & -2.281892 \\
\hline 38 & 1 & 0 & -2.654352 & -3.765655 & -1.583415 \\
\hline 39 & 1 & 0 & -4.106738 & -2.823981 & -1.829138 \\
\hline 40 & 1 & 0 & -3.415388 & -3.728564 & -3.154247 \\
\hline
\end{tabular}




\section{Optimized geometry of the complex}

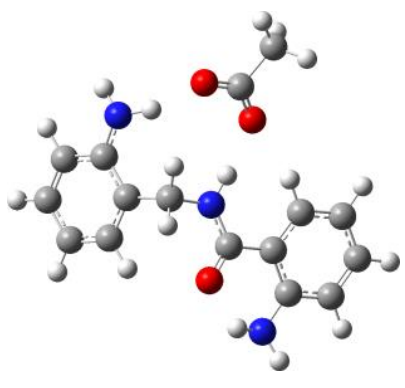

$E($ RB3LYP $)=-1010.93066797$ Hartree/particle

Standard orientation:

\begin{tabular}{|c|c|c|c|c|c|}
\hline \multirow{2}{*}{$\begin{array}{l}\text { Center } \\
\text { Number }\end{array}$} & \multirow{2}{*}{$\begin{array}{l}\text { Atomic } \\
\text { Number }\end{array}$} & \multirow{2}{*}{$\begin{array}{l}\text { Atomic } \\
\text { Type }\end{array}$} & \multicolumn{3}{|c|}{ Coordinates (Angstroms) } \\
\hline & & & $\mathrm{X}$ & $\mathrm{Y}$ & Z \\
\hline---- & & & & ---------1 & --ー-----ー \\
\hline 1 & 8 & 0 & 0.994611 & -1.941665 & 1.353397 \\
\hline 2 & 7 & 0 & 3.281217 & -2.748369 & 0.305265 \\
\hline 3 & 1 & 0 & 2.483184 & -2.901937 & 0.919106 \\
\hline 4 & 1 & 0 & 4.125524 & -3.255151 & 0.520630 \\
\hline 5 & 7 & 0 & 0.048734 & -0.027556 & 0.612507 \\
\hline 6 & 1 & 0 & 0.106629 & 0.894909 & 0.154416 \\
\hline 7 & 7 & 0 & -3.602945 & 0.955585 & 0.038488 \\
\hline 8 & 1 & 0 & -4.274726 & 1.368344 & -0.590103 \\
\hline 9 & 1 & 0 & -2.851649 & 1.615865 & 0.280848 \\
\hline 10 & 6 & 0 & 2.407593 & -0.462038 & 0.111751 \\
\hline 11 & 6 & 0 & 3.453423 & -1.415530 & -0.023036 \\
\hline 12 & 6 & 0 & 4.685427 & -0.998201 & -0.556782 \\
\hline 13 & 1 & 0 & 5.477855 & -1.733285 & -0.662896 \\
\hline 14 & 6 & 0 & 4.895097 & 0.310972 & -0.949823 \\
\hline 15 & 1 & 0 & 5.859375 & 0.600191 & -1.355229 \\
\hline 16 & 6 & 0 & 3.872182 & 1.247851 & -0.826817 \\
\hline 17 & 1 & 0 & 4.019551 & 2.275775 & -1.134634 \\
\hline 18 & 6 & 0 & 2.649160 & 0.854327 & -0.301595 \\
\hline 19 & 1 & 0 & 1.869959 & 1.601081 & -0.213395 \\
\hline 20 & 6 & 0 & 1.095498 & -0.862124 & 0.732524 \\
\hline 21 & 6 & 0 & -1.230263 & -0.317263 & 1.267690 \\
\hline 22 & 1 & 0 & -1.630821 & 0.637822 & 1.605208 \\
\hline 23 & 1 & 0 & -1.016125 & -0.928656 & 2.144593 \\
\hline 24 & 6 & 0 & -2.234513 & -1.045042 & 0.389823 \\
\hline 25 & 6 & 0 & -2.074592 & -2.412324 & 0.165397 \\
\hline 26 & 1 & 0 & -1.218994 & -2.898847 & 0.619604 \\
\hline 27 & 6 & 0 & -2.956495 & -3.145339 & -0.622324 \\
\hline 28 & 1 & 0 & -2.800342 & -4.205981 & -0.781221 \\
\hline 29 & 6 & 0 & -4.038605 & -2.487940 & -1.202135 \\
\hline 30 & 1 & 0 & -4.742449 & -3.032489 & -1.823657 \\
\hline 31 & 6 & 0 & -4.223355 & -1.131678 & -0.991524 \\
\hline 32 & 1 & 0 & -5.070918 & -0.625077 & -1.444453 \\
\hline 33 & 6 & 0 & -3.336506 & -0.378034 & -0.196177 \\
\hline 34 & 6 & 0 & -0.823446 & 3.438650 & 0.098438 \\
\hline 35 & 8 & 0 & -1.906170 & 3.132316 & 0.654978 \\
\hline 36 & 8 & 0 & 0.041215 & 2.641392 & -0.356975 \\
\hline 37 & 6 & 0 & -0.508293 & 4.934798 & -0.061621 \\
\hline 38 & 1 & 0 & 0.485870 & 5.149201 & 0.338108 \\
\hline 39 & 1 & 0 & -0.489319 & 5.185413 & -1.126114 \\
\hline 40 & 1 & 0 & -1.252771 & 5.552357 & 0.441431 \\
\hline
\end{tabular}


Theoretical IR and Raman spectra of acetate

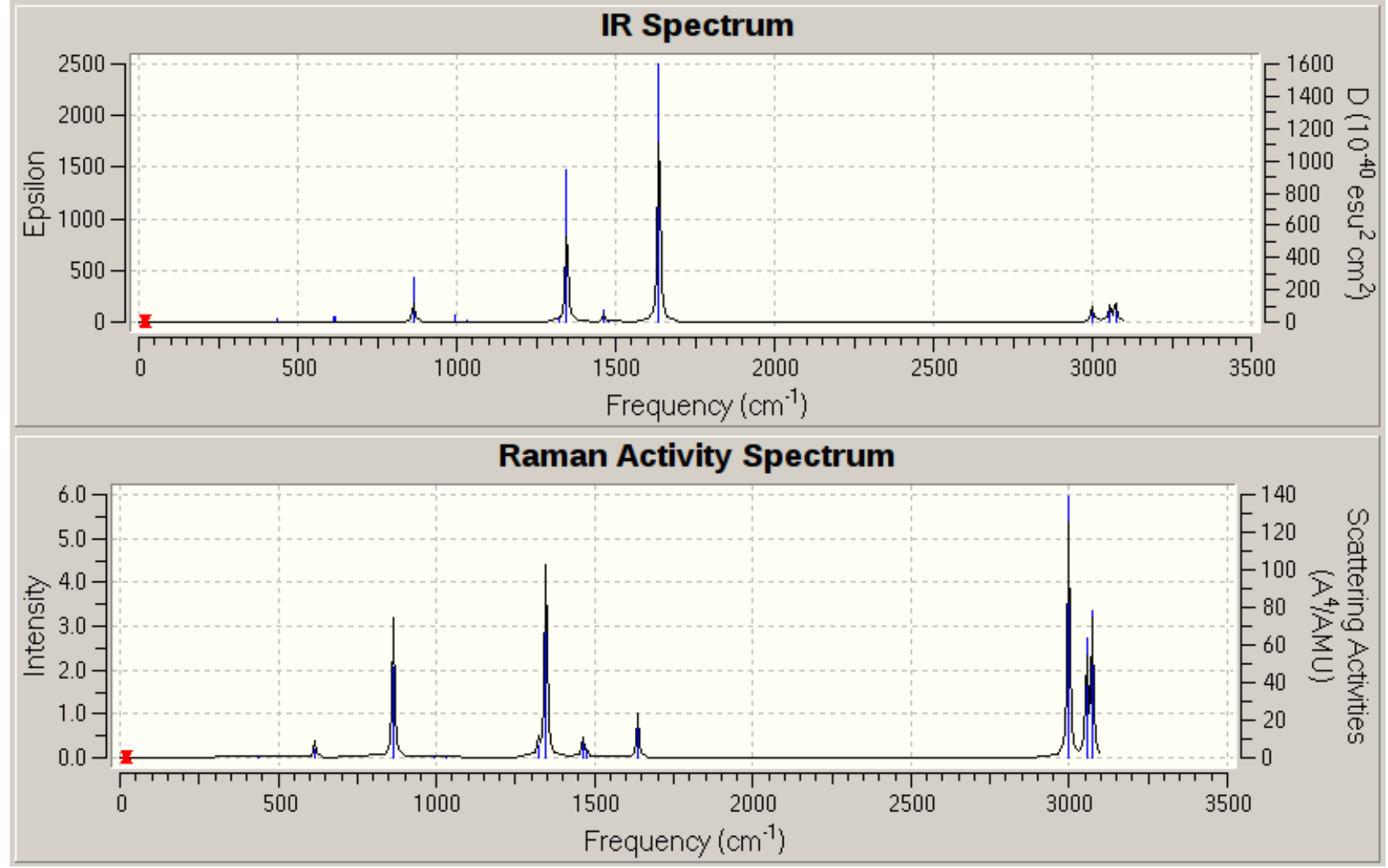

Theoretical IR and Raman spectra of the compound

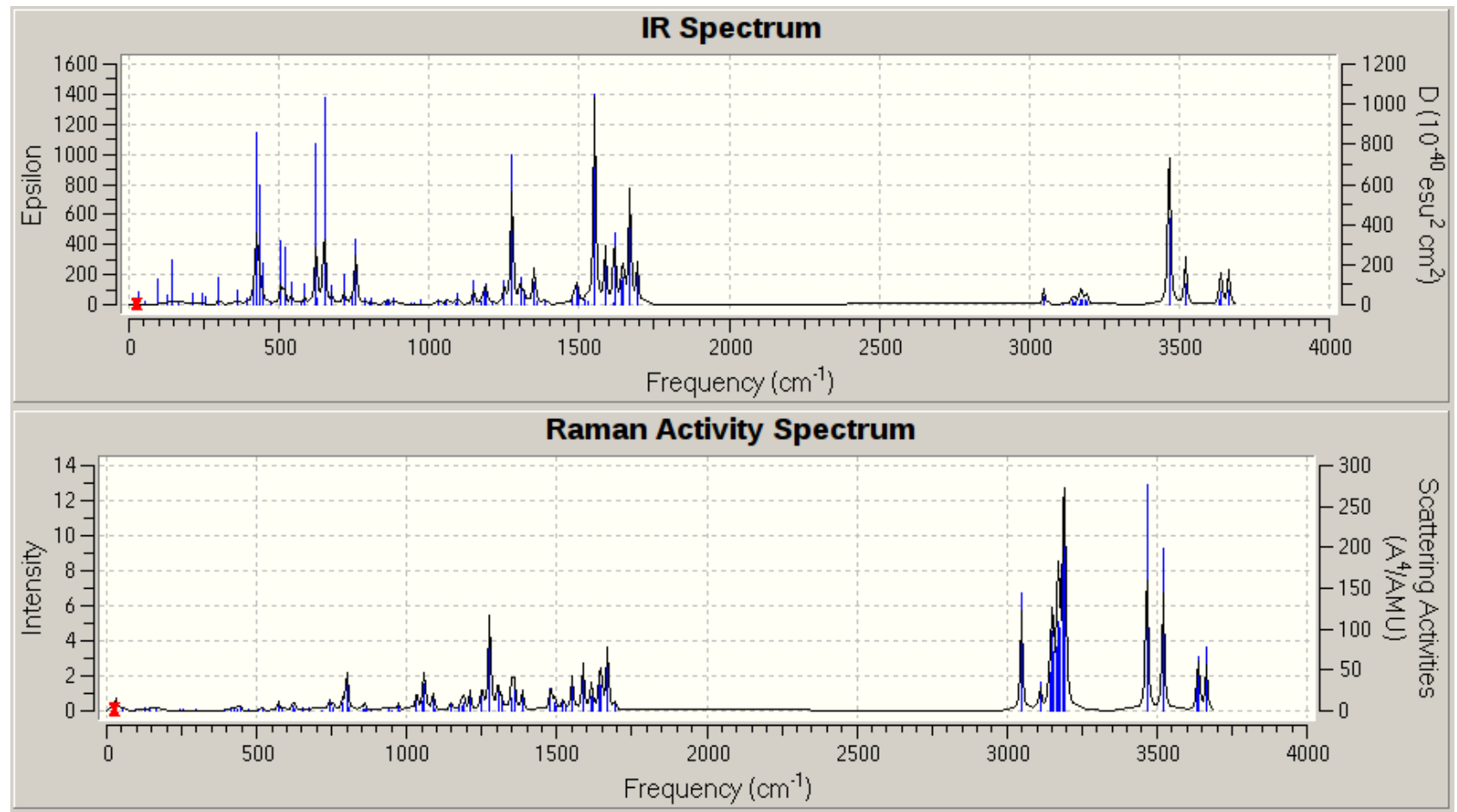


Theoretical IR and Raman spectra of the complex

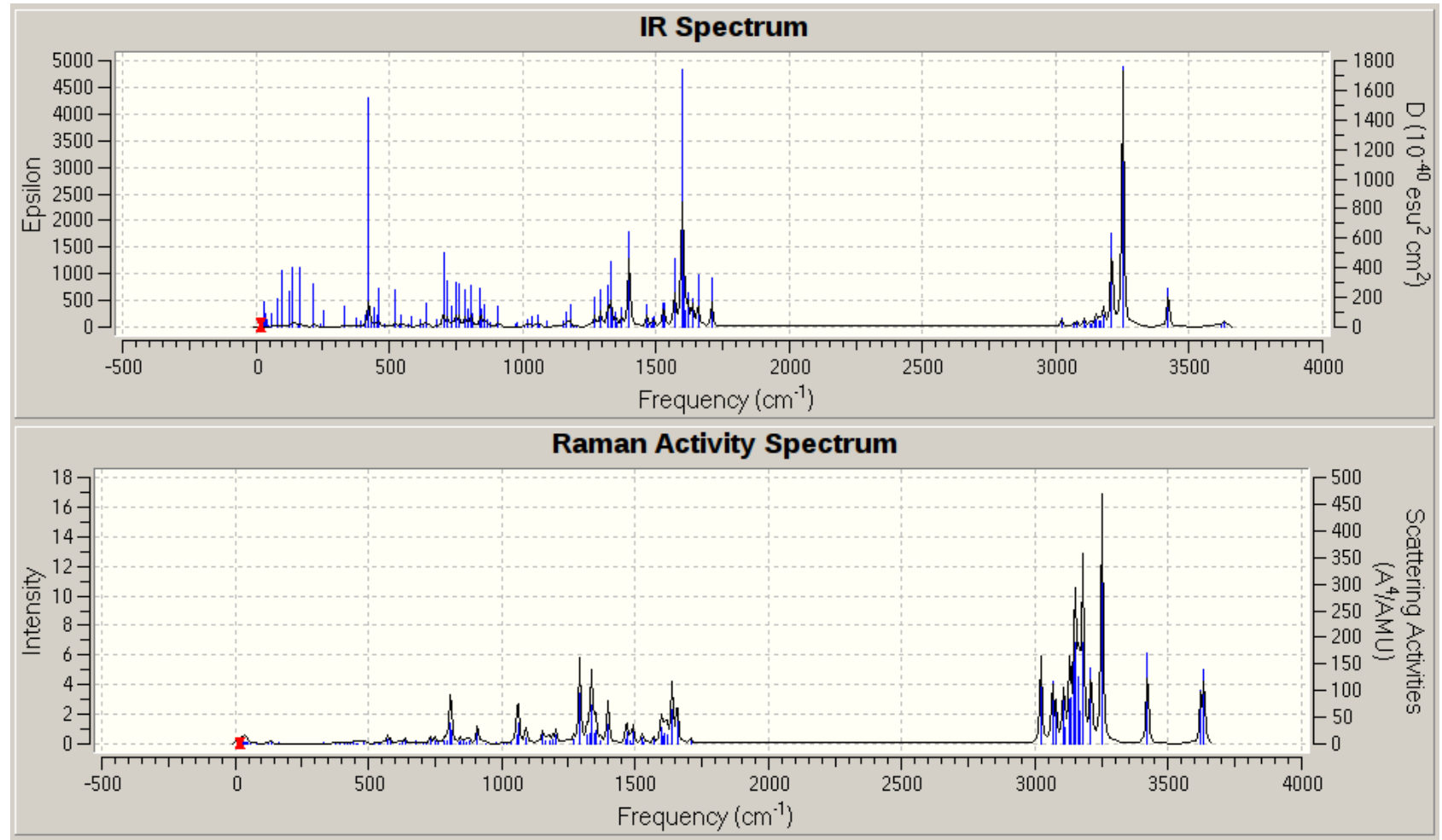

